\title{
The foot attack: Where are the defence mechanisms?
}

\author{
STELLA VIG, ${ }^{1}$ TALAL ALCHIKHAL ${ }^{1}$ AND BRIDGET TURNER, ${ }^{2}$ ON BEHALF OF DIABETES UK
}

\begin{abstract}
There is a need to raise awareness of foot complications and to decrease amputations in people with diabetes. The cost of care for these patients is high once they develop foot complications. With the correct management up to $80 \%$ of amputations are preventable, and decision makers are acknowledging that they can play an important role in the prevention and treatment of foot complications and thereby reduce the amputation rate. Commissioning high quality footcare and auditing standards of foot management pathways will be beneficial, as will prompt patient referral to a multidisciplinary footcare team. Signposting of these services to patients and carers may be the most important factor in preventing a minor foot problem escalating to an amputation.
\end{abstract}

Br J Diabetes Vasc Dis 2014;14:72-74

Key words: amputation, ulcers, foot, diabetes

\section{Introduction}

In 2001 the Diabetes National Service Framework announced 12 standards for the achievement of high quality diabetes care, consequent to which several documents have been published to assist in the attainment of these standards. The National Diabetes Support Team guide for the care of the diabetic foot considered screening, prevention, care pathways and multidisciplinary care provision to assist in strategic development and implementation to achieve high quality care. ${ }^{1}$ However the challenge remains. In addition to healthcare systems improving, patient awareness (eg regular self/carer inspection of feet) can prevent and reduce the progression of several conditions. Indeed, lack of sensation in the foot can result in unrecognised injury. ${ }^{2}$ Neuropathy is a precipitator of foot deformity as well as being the underlying cause of about $60 \%$ of foot ulcers. Peripheral vascular disease is a major contributor to $50 \%$ of foot ulcers and is associated with reduced healing and increased susceptibility to infection leading to gangrene and amputation. ${ }^{3}$ The commissioning of

Croydon University Hospital, Croydon, UK

Policy and Care Improvement, Diabetes UK, London, UK

Address for correspondence: Ms Stella Vig

Croydon University Hospital, 530 London Road, Croydon, CR7 7YE, UK.

Tel: +44 (0)208 4013403

E-mail: svig@doctors.org.uk

http://dx.doi.org/10.15277/bjdvd.2014.012

\author{
Abbreviations and acronyms \\ APPVG All Party Parliamentary Vascular Group \\ CCG Clinical Commissioning Groups \\ CLI critical limb ischaemia \\ MDfT multidisciplinary footcare team \\ NICE National Institute for Health and Care Excellence
}

high quality footcare and auditing standards of foot management pathways offer significant benefits, as does prompt patient referral to a multidisciplinary footcare team. ${ }^{4}$

In 2010-11, the NHS in England spent around $\mathrm{f} 650$ million on diabetic foot ulcers and amputation; this estimate equates to 0.6-0.7\% of its budget. ${ }^{5}$ In England $>6,000$ major amputations are carried out each year in patients with diabetes and, if the current rate continues, the number of amputations will rise to $>7,000$ in 2015/16. 6 People with diabetes are more likely to be admitted to hospital with a foot ulcer than with any other complication of diabetes. If infection and ischaemia are not addressed promptly, this may result in a minor or major amputation. Between 2006 and 2011 there has been a 46\% increase in amputations. ${ }^{7}$ Amputations and foot ulcers have a huge impact on quality of life and mortality remains high with up to $80 \%$ of people dying within 5 years of having an amputation. This is a higher mortality rate than colon, breast or prostate cancer, compared with which there is relatively little investment in public awareness and screening.

\section{Foot screening}

Feet are examined as part of the diabetes annual review and a foot risk status assigned. ${ }^{1}$ The examination should include a check for corns, calluses or changes in the shape of the feet as well as assessment of peripheral nerve function and pulses in the feet plus advice about foot care, including protection with correctly fitted footwear. Unfortunately, $15 \%$ of people with diabetes are not getting their annual foot check and there is variation between regions, ranging from $47-87 \%$ and $73-90 \%$ of people with type 1 and type 2 diabetes respectively ${ }^{7}$ (Figure 1). Diabetes UK has heard from patients who have supposedly received their foot check without being asked to remove their shoes! However alone the foot check is not enough as there is poor correlation between screening and amputation rates.

\section{Raising patient awareness}

Diabetes increases the risk of amputation 20-30 fold and amputation rates vary widely from one area to another - in the worst performing area a person with diabetes is ten times more likely 
Figure 1. Patients receiving annual foot checks ${ }^{7}$

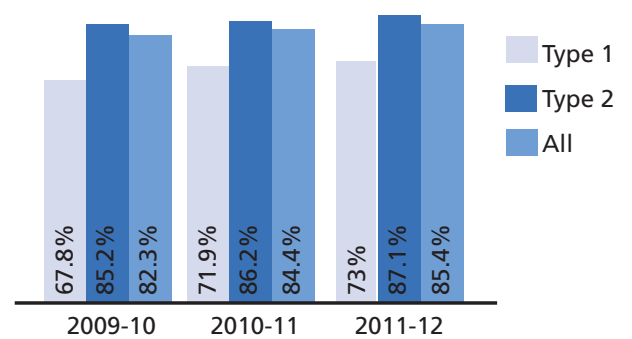

to have a major amputation than in the best.7,8

The footcare pathway appears to be key to facilitating best practice. About 15\% of patients with diabetes experience serious foot problems, and foot ulcers lead to about $85 \%$ of amputations. The ideal treatment pathway includes a 'foot protection team' and access to a multidisciplinary team within 24 hours of acute presentation with a foot complication. Surprisingly, more than half of people with diabetes surveyed in 2007 said that they did not realise that diabetes increased the risk of having an amputation. ${ }^{9}$ It is hoped that awareness has improved since then, but the key to improved awareness is placing patients with diabetes at the centre of their own care, as set out in the 'Year of Care' programme. ${ }^{10}$

In common with other diabetes-related complications, foot problems are associated with chronic sub-optimal control of modifiable risk factors - blood pressure, cholesterol and glucose levels and smoking. Good diabetes management and support for self-management, including smoking cessation, is important for the prevention of complications.

Patients should be empowered to look after their feet, told their risk of developing a complication, understand the implications of their risk status and be aware of the healthcare they should receive. It is crucial that patients realise the importance of urgently seeking medical attention in the event of any problems. A 'Touch the Toes' test guide is available on the Diabetes UK website and there is information about footcare, the leaflet '10 Steps to Healthy Feet' is also available in several languages.

\section{Quality training for staff}

There is a need for improved education and training for staff in primary and secondary care to facilitate provision of quality foot checks, risk status assessment, explanation of implications for the patient and appropriate referral pathways. Standards of care should be monitored nationally, and the impact on amputation rates measured. All staff should be encouraged to participate in diabetes audits. In the community there should be trained staff in foot protection services with speedy (<24hrs) access to MDfTs. The competency frameworks of these teams are documented in Diabetes UK's 'Putting Feet First' campaign located in the professional resources section of the Diabetes UK website. ${ }^{11}$

\section{Giving patients the tools}

The term 'foot attack' is used to describe a foot injury to a foot, or feet, of someone with diabetes who has neuropathy or peripheral vascular disease. Patients need to be aware that there may be no pain even with a visible wound and that even a small injury or blister may lead to a major complication unless arrested early.

Diabetes UK has produced a booklet called 'How to Spot a Foot Attack' for people who have been identified as being at risk. This booklet, which was sent to every GP surgery in England and Northern Ireland at the beginning of May, informs patients that they should have been referred to a foot protection team or specialist podiatrist, describes how to spot a 'foot attack' and what to do if they are experiencing one. The booklet also includes a card, where the person can write the emergency contact numbers of their GP, MDfT team and podiatry/foot care services.

The strength of a pathway and a multidisciplinary team is that the patients are triaged rapidly to the right subspeciality within the right timeframe. All patients with diabetes should know how to access these services if they develop a 'foot attack' and be encouraged to be insistent if their referral or treatment is delayed as a 'foot attack' can progress rapidly. ${ }^{12}$

\section{Equality in access}

In England and Wales the National Diabetes Audit measures the effectiveness of diabetes healthcare against the NICE Clinical Guidelines and NICE Quality Standards. This summer the National Foot Care Audit will commence an audit of specialist foot care services in England and Wales, aiming to highlight areas of good practice as well as areas which have not developed all elements of a high quality footcare pathway. This will provide benchmarking data to assist CCGs to commission excellent diabetic footcare services.

The Vascular Society of Great Britain and Ireland published a Quality Improvement Framework for Major Amputation Surgery recommending that patients should be managed pre-, peri- and post-operatively by a multidisciplinary vascular team with current amputation experience. ${ }^{13}$ The National Confidential Enquiry into Patient Outcomes and Deaths is due to publish its lower limb amputation study in Autumn 2014. This should inform on pre-, peri- and post-operative care as well as organisational factors. ${ }^{14}$ The Circulation Foundation has also raised awareness of the 'foot attack' with the 'Save Lives and Limbs' campaign as well as lobbying the APPVG. The APPVG has recently published recommendations which should improve patient outcomes (Table 1). ${ }^{15}$ Additionally, Strategic Clinical Networks have been tasked with raising awareness of and reducing major amputations by 2015.

\section{Conclusion}

An integrated structured footcare service between primary and specialist care is essential in reducing the risk of both minor and major amputations. Commissioners should be aware of the commissioned service within their area and aspects to be improved to reduce the cost of care for these high risk patients.

The current rate of major amputation is too high. It is vital 
Table 1 The All Party Parliamentary Vascular Group recommendations to improve patient outcomes

1. Services should be commissioned on outcomes: an amputation should be considered a failure; a functioning foot with minimal surgery, a success.

2. Telemedicine should link services so that appropriate care can be delivered locally with established pathway co-ordinators in hub centres and integrated clear pathways for the diabetic foot. There should be a named contact person in a hospital/community 24 hours a day who is a member of the MDfT in case of emergencies.

3. All commissioners should have a sub-24 hour policy to refer patients with suspected $\mathrm{CLI}$ to a MDfT. Time is of the essence with this condition, and every hour treatment is delayed increases the risk of amputation.

4. All commissioners and providers should have a clear pathway for suspected peripheral arterial disease and the diabetic foot. This pathway must be made standard practice, and the route that patients with CLI are referred to a hospital should be rapid, clear, and properly understood by all healthcare workers, from primary to specialist care. There should be a policy for referral to a multidisciplinary team with clear links to secondary care.

5. The Quality Outcomes Framework needs to be improved so that all 'high risk' patients are referred for preventative podiatry and structured education.

6. A patient pathway must be established as standard practice for all providers and commissioners.

7. Commissioning structures need to balance centralisation of care for complex high-risk vascular procedures with the need to maintain equity of patient access for peripheral arterial disease. This recognises that many diabetic foot complications occur in well perfused feet and do not need vascular intervention. Diabetic foot services therefore need to be aligned to the centralisation of vascular services but may not correlate completely.

8. Education for patients at risk should be made more widespread in the community. Guidance and support on smoking cessation and exercise, in particular for patients with diabetes, is one of the key areas that need attention.

that healthcare professionals understand the importance of performing good quality annual foot checks, providing patients with the tools they need to understand their risk of a 'foot attack' and, if necessary, enable patients to access specialist help as quickly as possible.

\section{Conflict of interest None Funding sources None}

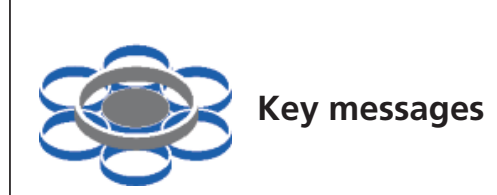

Diabetes patients should be:

- made aware of their foot risk and signposted to the Foot Protection Team.

- empowered to optimise self-care of the feet

- referred rapidly to a MDfT in the event of a 'foot attack'

Early intervention reduces complications and amputations.

\section{References}

1. Day C. Foot guide. Br J Diabetes Vasc Dis 2006;6:139-40.

2. Campbell IW. Diabetic foot disease - new thoughts on prevention and treatment. Br J Diabetes Vasc Dis 2011;11:53-54

3. Nag S, McCulloch A, Razvi S. Foot trauma due to foreign bodies - out of sight, out of mind? Br J Diabetes Vasc Dis 2004;6:421.

4. NHS Atlas of Variation in Healthcare (2010). NHS Right Care, http://www.rightcare.nhs.uk/atlas/downloads/EndocrineMaps_AoV_201 1.pdf (Accessed May 2014).

5. Kerr M. Foot Care for People with Diabetes: The Economic Case for Change. Insight Health Economics. March 2012. https://www. diabetes.org.uk/Documents/nhs-diabetes/footcare/footcare-for-people-with-diabetes.pdf (Accessed May 2014).

6. The Information Centre for Health and Social Care. Hospital Episode Statistics 2007/08-2010/11 http://www.hscic.gov.uk/ (Accessed May 2014)

7. State of the Nation. England. 2013. http://www. diabetes.org.uk/Documents/About\%20Us/What\%20we\%20say/0160b-state-nation-2013england-1213.pdf (Accessed May 2014).

8. Holman N, Young RJ, Jeffcoate WJ. Variation in the recorded incidence of amputation of the lower limb in England. Diabetologia 2012;55:1919-25.

9. Ipsos MORI. Survey on awareness of Diabetes and Diabetes UK amongst the general public, conducted on behalf of Diabetes UK 2007.

10. Year of care. Report of findings from the pilot programme. June 2011 http://www.diabetes.org.uk/upload/Professionals/Year\%20of\%20Care/ YOC Report.pdf (Accessed May 2014).

11. Putting feet first. Commissioning specialist services for the management and prevention of diabetic foot disease in hospital. June 2009 http://www.diabetes.org.uk/Documents/Reports/Putting_Feet_First_010 709.pdf (Accessed May 2014).

12. Tobalem M, Uckay I. Evolution of a diabetic foot infection. New Engl Med 2013:369:2252.

13. Vascular Society of Great Britain and Ireland. Quality Improvement Framework for Major Amputation Surgery. March 2012 http://www.vascularsociety.org.uk/doc-category/audit-qi/ (Accessed May 2014).

14. The National Confidential Enquiry into Patient Outcomes and Deaths. http://www.ncepod.org.uk/amputation.htm (Accessed May 2014).

15. All Party Parliamentary Group on Vascular Disease. Tackling peripheral arterial disease more effectively: saving limbs, saving lives. http://appgvascular.org.uk/media/reports/2014-03-tackling_peripheral_arterial disease_more_effectively_saving_limbs_saving_lives.pdf (Accessed May 2014) 